\title{
Circadian regulation of renal function and potential role in hypertension.
}

\author{
Olivier Bonny ${ }^{1,2 \#}$ and Dmitri Firsov ${ }^{1 \#}$
}

${ }^{1}$ - Department of Pharmacology and Toxicology, University of Lausanne, Switzerland

2 - Service of Nephrology, Department of Medicine, CHUV, Lausanne, Switzerland

\# - to whom correspondence should be addressed:

Dmitri Firsov, Department of Pharmacology and Toxicology, University of Lausanne, 27 rue du Bugnon, 1005 Lausanne, Switzerland. e-mail: dmitri.firsov@unil.ch

Phone: ++41216925406

and

Olivier Bonny, Department of Pharmacology and Toxicology, University of Lausanne, 27 rue du Bugnon, 1005 Lausanne, Switzerland. e-mail: olivier.bonny@unil.ch Phone: ++41216925417

The authors have declared that no conflict of interest exists.

Key points: 1 . The circadian clock controls a large number of specific renal functions. 2. The circadian clock is involved in renal sodium handling through a variety of recently elucidated mechanisms. 3. Alteration of circadian rhythms in humans is associated with several conditions, including decreased glucose sensitivity, hypertension and cancer. 4 .

Chronopharmacotherapy is an emerging field that may help treating conditions like hypertension and cancer. 
Purpose of review: Previous studies have shown that a variety of specific renal functions exhibit circadian oscillations. This review aims (i) to provide an update on the molecular mechanisms underlying circadian rhythms in the kidney, and (ii) to discuss how dysregulation of circadian rhythms can interfere with kidney function.

Recent findings: The molecular mechanism responsible for generating and maintaining circadian rhythms has been unraveled in great detail. This mechanism, known as circadian clock, drives circadian oscillation in expression levels of a large number of renal mRNA transcripts. Several proteins critically involved in renal homeostatic functions have been shown to exhibit significant circadian oscillation in their expression levels or in their posttranslational modifications. In transgenic mouse models, disruption of circadian clock activity results in dramatic changes in the circadian pattern of urinary sodium and potassium excretion and causes significant changes in arterial blood pressure. A growing number of evidence suggests that dysregulation of circadian rhythms is associated with the development of hypertension and accelerated progression of chronic kidney disease (CKD) and cardiovascular disease in humans. Chronotherapy studies have shown that the efficacy of antihypertensive medication is greatly dependent on the circadian time of drug administration. Summary: Recent research points to the major role of circadian rhythms in renal function and in control of blood pressure.

Keywords: kidney, circadian clock, sodium, blood pressure, chronopharmacology. 


\section{Introduction}

Circadian rhythmicity of renal function is a long-studied phenomenon which was first documented in the middle of the $19^{\text {th }}$ century when Edward Smith and other pioneers in chronobiology noted that urinary water and urea excretion exhibit significant circadian oscillations [1]. The existence of circadian rhythms was subsequently revealed in a number of specific renal functions. Despite the apparent correlation of renal circadian rhythms with rhythms of activity and feeding, physiological studies demonstrated that several important rhythmic processes in the kidney (e.g. circadian rhythm of urinary potassium excretion) are largely self-sustained and intrinsic in origin (reviewed in [2,3]). On the molecular level, it has been shown that circadian rhythms are primarily driven by the circadian clock, a complex mechanism that tightly coordinates all major cellular functions with the time of the 24-hour geophysical cycle (circadian time). This coordination provides an important functional advantage which consists in time-of-day-dependent preconditioning of cells and organs to recurrent environmental circadian changes.

Long-term misalignment of intrinsic circadian rhythms with environmental time has been shown as an independent risk factor for the progression of a variety of diseases, including diabetes, cardiovascular disease, chronic kidney disease and cancer (reviewed in $[4,5])$. Genome Wide Association Studies (GWAS) have suggested a genetic link between single nucleotide polymorphisms (SNPs) in genes involved in the circadian clock and type 2 diabetes [6], hypertension [7] and mood disorders [8]. A growing number of mouse models in which the activity of the circadian clock was modified have demonstrated that this mechanism is indispensable for many vital processes, including renal homeostatic function and blood pressure control. In this review we summarize advances achieved within the last 12-18 months in the understanding of the role of the circadian timing system in kidney health and disease. 
by a highly complex mechanism operating at different molecular levels. The first discovered and most studied circadian clock mechanism involves the transcriptional factors BMAL1, CLOCK and NPAS2 and their repressors PER and CRY (reviewed in [**9]). These proteins constitute the core of a transcriptional/translational feedback loop that generates transcriptional rhythms with a periodicity of $\sim 24$ hours. The BMAL1/CLOCK or BMAL1/NPAS2 heterodimers activate transcription and translation of PER and CRY during the daytime. Accumulation of PER and CRY proteins in the cytoplasm results in their heterodimerization and translocation into the nucleus where PER/CRY complexes bind to and inhibit the transcriptional activity of BMAL1/CLOCK and BMAL1/NPAS2. In the absence of de novo synthesis, PER and CRY are progressively depleted during the night by the ubiquitination/proteosome degradation pathway, thus resulting in the relief of the transcriptional repression (so completing the 24-hour cycle). Activity of this circadian clock in peripheral tissues is largely self-sustained and cell-autonomous, but its synchronization with the environmental time is dependent on a central pacemaker located in the suprachiasmatic nucleus of hypothalamus. In addition to the regulation of PER and CRY transcription, the BMAL1/CLOCK and BMAL1/NPAS2 heterodimers are thought to generate circadian oscillations of $2-10 \%$ of cellular transcriptome. For instance, Zuber et al. have shown that hundreds of mRNA transcripts in the microdissected distal convoluted tubule (DCT), connecting tubule (CNT) and cortical collecting duct (CCD) exhibit significant circadian oscillations, and Nikolaeva et al. have demonstrated that circadian rhythmicity of many of these transcripts is abolished in mice devoid of CLOCK [10,**11]. Many among the oscillating transcripts are crucially involved in different homeostatic renal functions e.g., 
aquaporin 2- and 4- water channels, the V2 vasopressin receptor, the NHE3 sodium proton exchanger, serum- and glucocorticoid- induced kinase 1 (Sgk1), the H,K-ATPase type 2 and the regulator of the Na,K-ATPase Fxyd5 [10,12,*13,14].

This classical model of the circadian clock based on the transcriptional oscillations has been seriously challenged by several recent studies. Menet et al. and Koike et al. have shown that oscillations of only about $20 \%$ of mature circadian mRNAs result from the circadian transcription $[15, * * 16]$. These studies pointed to the very important role of posttranscriptional events in the generation of circadian transcriptome. Another level of complexity in the circadian clock was revealed by the discovery of so-called "metaclocks" involving antioxidant proteins peroxiredoxins (PRX). PRXs exhibit transcription-independent oscillations in their oxidation states that parallel circadian production of reactive oxygen species (ROS) in mitochondria. Importantly, Edgar et al. have shown that the PRX oxidation state can influence the circadian transcriptional component, thereby demonstrating the interconnectivity between different circadian mechanisms [*17]. Finally, Jouffe et al. have recently demonstrated that the circadian clock controls ribosome biogenesis, a mechanism which is thought to be involved in the dynamic regulation of energy homeostasis in the cell [18].

Circadian rhythms in the kidney Accumulating evidence suggests that most if not all specific renal functions exhibit circadian oscillations. For instance, many renal rhythms are imposed by the circadian rhythmicity in the glomerular filtration rate (GFR), the renal blood flow (RBF) and the plasma concentration of filtered solutes. As an example, both the GFR and the plasma concentration of amino acids rise by $\sim 50 \%$ during the activity phase of the circadian cycle, thereby generating significant circadian oscillations in the filtered load of amino acids (cumulative amplitude of $\sim 100 \%$ ) [19]. This, in turn, imposes a requirement for circadian adaptation in the reabsorption capacity for amino acids in the proximal tubule. How does this adaptation occurs at the molecular level has not been investigated, but our analyses 
of renal circadian transcriptomes has shown that several major apical and basolateral transporters of amino acids exhibit significant circadian oscillations in their mRNA expression levels (Figure 1). A similar rationale can be applied to a wide variety of filtered substances since Dallmann et al. and Eckel-Mahan et al. have recently shown that at least $15 \%$ of blood metabolites cycle with a 24 -hour rhythmicity (this rationale is depicted in Figure 2) $\left[* 20,{ }^{*} 21\right]$.

Previous functional studies have suggested that the circadian clock is involved in blood pressure control. For instance, Bankir et al. demonstrated that the circadian dynamic of urinary sodium excretion is an important determinant of dipping in nocturnal blood pressure [22]. The discovery of the clock genes has allowed a direct assessment of the role of the circadian clock in renal function and in blood pressure control. Woon et al. have identified several polymorphisms in the promoter region of the human $B M A L 1$ gene that are associated with hypertension [7]. Mice with targeted disruption of any of the positive or negative elements of the circadian clock based on the transcriptional feedback loop display either decreased or increased blood pressure (reviewed in [2,23]). Recent studies have begun to shed light on the possible role of the kidney in these dysfunctions. As mentioned above, a great number of renal mRNA transcripts exhibit circadian expression pattern. However, only a few proteins have been experimentally shown to undergo circadian oscillations. For instance, Saifur Rohman et al. have shown that mRNA and protein expression of sodium-proton exchanger 3 (NHE3) displays a circadian expression pattern which was blunted in CRY1/CRY2 double knockout mice [24]. Recently, Susa et al. have demonstrated that proteins involved in renal sodium homeostasis and blood pressure control can be modified in a circadian manner on the posttranslational level [**25]. By analyzing WNK-OSR1/SPAKNCC signaling cascade they were able to show that the phosphorylation level of OSR 1 and SPAK kinases as well as the sodium-chloride co-transporter (NCC) display significant 
circadian oscillation with the peak of phosphorylation at the time of transition between the inactive and active phases of the circadian cycle. The authors also demonstrate that this rhythmicity in phosphorylation state is driven by aldosterone and they interpreted the circadian phosphorylation kinetic of the cascade as a requirement to decrease sodium reabsorption during the active phase and to increase during the rest phase.

Aldosterone is the major regulator of sodium balance and blood pressure. Doi et al. have shown that secretion of this mineralocorticoid hormone by the adrenal glands is controlled by the circadian clock [26]. The authors demonstrated that beta-hydroxyl-steroid dehydrogenase (Hsd3b6), a steroidogenic enzyme involved in aldosterone biosynthesis, is transcriptionally regulated by the molecular clock. In Cry1/Cry2 double knockout mice that exhibit constantly elevated activity of the circadian clock, the plasma aldosterone levels are markedly increased causing salt-sensitive hypertension. Gumz and colleagues have shown that aldosterone can influence sodium reabsorption in the distal nephron and the collecting duct by interfering with the circadian clock activity. They demonstrated that aldosterone upregulates the expression level of the alpha subunit of the epithelial sodium channel $(\alpha \mathrm{ENaC})$ in part through the stimulation of Per1 expression $[27,28]$. This finding was recently supported and extended by Richards et al. who demonstrated that $\alpha \mathrm{ENaC}$ expression and $\mathrm{ENaC}$ activity are significantly reduced upon inhibition of casein kinases $1 \delta$ and $1 \varepsilon(\mathrm{CK} 1 \delta / \varepsilon)$ which are required for the phosphorylation and nuclear localization of PER/CRY heterodimers [*29]. The same group has recently reported that Per1 knockout mice exhibit low blood pressure [12].

Recently, we have shown that mice devoid of CLOCK exhibit dramatic changes in the circadian dynamic of urinary sodium, potassium and water excretion [**11]. Further functional analysis revealed that suppression of CLOCK abolishes the normal circadian rhythmicity of plasma aldosterone levels and results in a significant reduction in blood 
pressure. Importantly, all these changes occurred despite normal pattern of physical activity and water intake in the knockout mice. To identify molecular mechanism underlying these dysfunctions we analyzed transcriptional changes in kidneys of these mice. Interestingly, the most significant difference was observed in the expression levels of cytochrome-P450 enzymes (Cyp4a12 and Cyp4a14) involved in the oxidation of arachidonic acid to 20Hydroxyeicosatetraenoic acid (20-HETE). Analysis of temporal changes in 20-HETE levels in the kidney revealed a circadian-like pattern that was significantly modified in the knockout mice. 20-HETE has been shown to have a complex influence on blood pressure by inducing vasoconstriction and by inhibiting sodium reabsorption in the renal tubule. Collectively, this study suggested that the Cyp4a/20-HETE pathway could be one of the regulatory mechanisms by which the circadian clock participates in blood pressure control.

The circadian clock and human diseases. Given the pivotal role of the circadian clock in a variety of cellular functions, numerous studies have addressed the role of the circadian clock genes in pathogenesis of human diseases. Different animal models have been created and/or used to evaluate this issue. One of the most striking finding was obtained in the model of golden hamster heterozygous for a loos-of-function mutation in the circadian regulatory gene casein kinase-1epsilon [30]. These animals exhibit extensive fibrosis leading to cardiomyopathy, renal insufficiency and premature death when exposed to the light-dark cycles longer than the period of their intrinsic circadian clock, while the same animals kept in the light-dark cycle synchronized with their intrinsic circadian clock do perfectly well [31]. Another example is given by mice devoid of the three clock output genes DBP-HLF-TEF which display lower blood pressure, lower aldosterone levels and cardiac hypertrophy [32]. In humans, evidence showing a link between the circadian clock and diseases is weaker and more indirect. Unbiased genome-wide association studies (GWAS) have associated single nucleotide polymorphisms in loci close to genes involved in the molecular 
clock with several traits. When searched in the catalog of published GWAS

(http://www.genome.gov/gwastudies/), we found that rs900145, close to BMAL1 (ARNTL), was associated with age at menarche [33] and plasminogen activator inhibitor-1 (PAI-1) plasma levels [34], and that the CRY2 variant rs11605924 was associated with fasting glucose levels and beta cell function [6,35]. Moreover, a CLOCK variant was associated with agreeableness in one study [8] and NPAS2 with a variant form of Creutzfeldt-Jakob disease in another study [36]. The PER1, PER2, CRY1, DBP, TEF and $H L F$ or other genes coding for important components of the molecular clock have not display any significant association in GWAS conducted so far. In order to establish causality, one would need to study human beings with proven and unique deletion or mutation leading to significant disruption of the circadian clock. The only Mendelian disease known to be associated with the circadian clock is the autosomal dominant familial advanced sleep-phase syndrome (OMIM 604348) in which the genes PER2 and CSNK1D are mutated [37-39]. However, these families are extremely rare, poorly characterized and no data on their long-term health status is available. Another rare syndrome recently associated with the circadian clock function is the Smith-Magenis syndrome in which haploinsufficiency of RAIl gene leads to a complex clinical phenotype characterized by low intellectual capabilities, sleep-disorders disturbances, obesity and various neurobehavioral and congenital anomalies. One of the hallmarks of this syndrome is an inverted melatonin rhythm that leads, as a consequence, to diurnal somnolence and nocturnal sleep difficulties. RAI1 was shown to be an important regulator of the expression of CLOCK and other components of the clock system [40].

One of the most prominent circadian traits in human is blood pressure. Nocturnal dip of blood pressure is a well-known phenomenon, but the underlying physiological mechanism(s) still remains largely elusive. Numerous studies have attempted to address the role of the circadian clock in this phenomenon in shiftworkers [41-44]. These studies, 
however, have yielded in conflicting results. In total, evidence establishing causality between shiftwork and hypertension are scarce and do not allow definitive conclusion.

Chronotherapy for hypertension? Accumulating evidence suggests that the antihypertensive medication should be preferentially taken at vesper time. Using HYGIA cross-sectional data and MAPEC prospective data, Hermida and colleagues showed that prescription of at least one anti-hypertensive drug at bedtime controlled hypertension significantly better than when taking the treatment exclusively in the morning [45]. These and other data were recently reviewed in a meta-analysis of 21 studies that included $\sim 2000$ patients suffering from primary hypertension [46]. Evening taking of antihypertensive drugs was associated with a significantly better control of 24 hour blood pressure than morning dosing (SBP: $-1.71 \mathrm{~mm} \mathrm{Hg}, 95 \% \mathrm{CI}:-2.78$ to -0.65 ; DBP: $-1.38 \mathrm{~mm} \mathrm{Hg}$, 95\% CI: -2.13 to 0.62), but the clinical relevance and the effect on harder endpoints such as cardiovascular events remains unexplored. The mechanisms by which this effect would take place are still speculative. Pharmacokinetics and/or pharmacodynamics profiles for a given drug might be more optimal in the evening, even though almost any kind of antihypertensive drug tested so far has shown better effect when taken in the evening. This suggests a more general, drugindependent mechanism.

\section{Conclusions}

Identification of the molecular components of the clock system has allowed significant advances in the comprehension of circadian rhythms at the transcriptional level, and has brought forward new mechanisms of regulation e.g. at translational level or by redox reaction. The renal and hypertension fields start to benefit from these progresses. In particular, circadian rhythms should be taken into account in daily interpretation of data collected at 
different times of the day. Research should be oriented at deciphering circadian rhythms of complex traits i.e. traits in which several levels of circadian-dependent regulation are taking place. Chronotherapeutics may help ameliorating the control of blood pressure in humans and open new perspective in other fields.

\section{Figure legend.}

Figure 1. Circadian expression patterns of mRNAs encoding different amino acid (AA) transporters in the mouse proximal tubule. $\mathrm{Y}$-axis is the expression level in arbitrary units. ZT

- Zeitgeber time units: ZT0 is the time of light on and ZT12 is the time of light off.

Figure 2. Different origin of circadian rhythms in the kidney. The circadian rhythmicity in filtered loads results from the circadian rhythms in the GFR, the RBF and the circadian oscillations in blood levels of filtered compounds. The circadian rhythms in urinary excretion is the sum of circadian rhythms in filtered loads and tubular reabsorption/secretion. Of note, the period length of these functional rhythms is approximately the same ( $\sim 24$ hours), but the difference between their maximum and minimum values (amplitude) as well as the time of the day when the given process function at a maximal rate (acrophase) can vary considerably.

ACKNOWLEDGMENTS This work was supported by the Swiss National Science Foundation research grants 31003A-132496 (to D.F.) and PP00P3-133648/1 (to O.B.).

1. Smith E (Ed): Health and disease as influenced by the daily, seasonal, and other cyclical changes in the human system. London: Walton and Maberly; 1861.

2. Firsov D, Bonny O. Circadian regulation of renal function. Kidney International 2010, 78:640-645.

3. Firsov D, Tokonami N, Bonny O. Role of the renal circadian timing system in maintaining water and electrolytes homeostasis. Mol Cell Endocrinol 2012, 349:51-55.

4. Maury E, Ramsey KM, Bass J. Circadian rhythms and metabolic syndrome: from experimental genetics to human disease. Circ Res 2010, 106:447-462.

5. Kimura G, Dohi Y, Fukuda M. Salt sensitivity and circadian rhythm of blood pressure: the keys to connect CKD with cardiovascular events. Hypertens Res 2010, 33:515-520. 
6. Manning AK, Hivert MF, Scott RA, et al. A genome-wide approach accounting for body mass index identifies genetic variants influencing fasting glycemic traits and insulin resistance. Nat Genet 2012, 44:659-669.

7. Woon PY, Kaisaki PJ, Braganca J, et al. Aryl hydrocarbon receptor nuclear translocator-like (BMAL1) is associated with susceptibility to hypertension and type 2 diabetes. Proc Natl Acad Sci $U S A$ 2007, 104:14412-14417.

8. Terracciano A, Sanna S, Uda M, et al. Genome-wide association scan for five major dimensions of personality. Mol Psychiatry 2010, 15:647-656.

**9. Bass J. Circadian topology of metabolism. Nature 2012, 491:348-356.

This review summaraizes recent progeress in understanding the mechanisms underlying circadian rhythms.

10. Zuber AM, Centeno G, Pradervand S, et al. Molecular clock is involved in predictive circadian adjustment of renal function. Proc Natl Acad Sci $U S A$ 2009, 106:16523-16528.

**11. Nikolaeva S, Pradervand S, Centeno G, et al. The circadian clock modulates renal sodium handling. J Am Soc Nephrol 2012, 23:1019-1026.

This study illustrates for the first time the role of the circadian clock in renal sodium handling.

12. Stow LR, Richards J, Cheng KY, et al. The circadian protein period 1 contributes to blood pressure control and coordinately regulates renal sodium transport genes. Hypertension 2012, 59:11511156.

*13. Salhi A, Centeno G, Firsov D, et al. Circadian expression of H,K-ATPase type 2 contributes to the stability of plasma K(+) levels. FASEB J 2012, 26:2859-2867.

This study demonstrates that the H,K-ATPase type 2 contributes to the circadian rhythm of urinary potassium excretion.

14. Saifur Rohman M, Emoto N, Nonaka H, et al. Circadian clock genes directly regulate expression of the $\mathrm{Na}(+) / H(+)$ exchanger NHE3 in the kidney. Kidney Int 2005, 67:1410-1419.

15. Menet JS, Rodriguez J, Abruzzi KC, et al. Nascent-Seq reveals novel features of mouse circadian transcriptional regulation. elife 2012, 1:e00011.

**16. Koike N, Yoo SH, Huang HC, et al. Transcriptional architecture and chromatin landscape of the core circadian clock in mammals. Science 2012, 338:349-354.

This study highlights the importance of post-transcriptional events in the generation of circadian transcriptome.

*17. Edgar RS, Green EW, Zhao Y, et al. Peroxiredoxins are conserved markers of circadian rhythms. Nature 2012, 485:459-464.

This study describes the role of oxidation-reduction cycles of peroxiredoxin proteins in the genesis of the ciracdain rhythms.

18. Jouffe C, Cretenet G, Symul L, et al. The circadian clock coordinates ribosome biogenesis. PLoS Biol 2013, 11:e1001455.

19. Feigin RD, Klainer AS, Beisel WR. Circadian periodicity of bloof amino-acids in adult men. Nature 1967, 215:512-514.

*20. Dallmann R, Viola AU, Tarokh L, et al. The human circadian metabolome. Proc Natl Acad Sci U S A 2012, 109:2625-2629.

Together with the paper by Eckel-Mahan et al. (21), this study shows that dozens of metabolites exhibit circadian rhythmicity in their plasma levels. This observation highlights significant ciracdian oscillations in fileters loads for many, if not all, substances.

*21. Eckel-Mahan KL, Patel VR, Mohney RP, et al. Coordination of the transcriptome and metabolome by the circadian clock. Proc Natl Acad Sci U S A 2012, 109:5541-5546.

Together with the paper by Dallmann et al. (20), this study shows that dozens of metabolites exhibit circadian rhythmicity in their plasma levels. This observation highlights significant ciracdian oscillations in fileters loads for many, if not all, substances.

22. Bankir L, Bochud M, Maillard M, et al. Nighttime blood pressure and nocturnal dipping are associated with daytime urinary sodium excretion in African subjects. Hypertension 2008, 51:891898.

23. Richards J, Gumz ML. Advances in understanding the peripheral circadian clocks. FASEB J 2012, 26:3602-3613.

24. Saifur Rohman M, Emoto N, Nonaka H, et al. Circadian clock genes directly regulate expression of the $\mathrm{Na}(+) / \mathrm{H}(+)$ exchanger NHE3 in the kidney. Kidney Int 2005, 67:1410-1419.

**25. Susa K, Sohara E, Isobe K, et al. WNK-OSR1/SPAK-NCC signal cascade has circadian rhythm dependent on aldosterone. Biochem Biophys Res Commun 2012, 427:743-747. 
This is the first study demonstrating the circadian rhythmicity in posttranslational modifications of proteins involved in renal sodium and potassium handling.

26. Doi M, Takahashi Y, Komatsu R, et al. Salt-sensitive hypertension in circadian clock-deficient Crynull mice involves dysregulated adrenal Hsd3b6. Nat Med 2009, 16:67-74.

27. Gumz ML, Stow LR, Lynch IJ, et al. The circadian clock protein Period 1 regulates expression of the renal epithelial sodium channel in mice. J Clin Invest 2009, 119:2423-2434.

28. Gumz ML, Cheng KY, Lynch IJ, et al. Regulation of alphaENaC expression by the circadian clock protein Period 1 in mpkCCD(c14) cells. Biochim Biophys Acta 2010, 1799:622-629.

*29. Richards J, Greenlee MM, Jeffers LA, et al. Inhibition of alphaENaC expression and ENaC activity following blockade of the circadian clock-regulatory kinases CK1delta/\{varepsilon\}. Am J Physiol Renal Physiol 2012, 303:F918-927.

This study describes the molecualr mechansisms by which the circadian clock controls the activity of the epithelail sodiumc hannel $(\mathrm{ENaC})$.

30. Ralph MR, Menaker M. A mutation of the circadian system in golden hamsters. Science 1988, 241:1225-1227.

31. Martino TA, Oudit GY, Herzenberg AM, et al. Circadian rhythm disorganization produces profound cardiovascular and renal disease in hamsters. Am J Physiol Regul Integr Comp Physiol 2008, 294:R1675-1683.

32. Wang Q, Maillard M, Schibler U, et al. Cardiac hypertrophy, low blood pressure, and low aldosterone levels in mice devoid of the three circadian PAR bZip transcription factors DBP, HLF, and TEF. Am J Physiol Regul Integr Comp Physiol 2010, 299:R1013-1019.

33. Elks CE, Perry JR, Sulem P, et al. Thirty new loci for age at menarche identified by a meta-analysis of genome-wide association studies. Nat Genet 2010, 42:1077-1085.

34. Huang J, Sabater-Lleal M, Asselbergs FW, et al. Genome-wide association study for circulating levels of PAI-1 provides novel insights into its regulation. Blood 2012, 120:4873-4881.

35. Dupuis J, Langenberg C, Prokopenko I, et al. New genetic loci implicated in fasting glucose homeostasis and their impact on type 2 diabetes risk. Nat Genet 2010, 42:105-116.

36. Sanchez-Juan P, Bishop MT, Aulchenko YS, et al. Genome-wide study links MTMR7 gene to variant Creutzfeldt-Jakob risk. Neurobiol Aging 2012, 33:1487 e1421-1488.

37. Jones CR, Campbell SS, Zone SE, et al. Familial advanced sleep-phase syndrome: A short-period circadian rhythm variant in humans. Nat Med 1999, 5:1062-1065.

38. Toh KL, Jones CR, He Y, et al. An hPer2 phosphorylation site mutation in familial advanced sleep phase syndrome. Science 2001, 291:1040-1043.

39. Xu Y, Padiath QS, Shapiro RE, et al. Functional consequences of a CKIdelta mutation causing familial advanced sleep phase syndrome. Nature 2005, 434:640-644.

40. Williams SR, Zies D, Mullegama SV, et al. Smith-Magenis syndrome results in disruption of CLOCK gene transcription and reveals an integral role for RAI1 in the maintenance of circadian rhythmicity. Am J Hum Genet 2012, 90:941-949.

41. Boggild H, Knutsson A. Shift work, risk factors and cardiovascular disease. Scand J Work Environ Health 1999, 25:85-99.

42. Hublin C, Partinen M, Koskenvuo K, et al. Shift-work and cardiovascular disease: a population-based 22-year follow-up study. Eur J Epidemiol 2010, 25:315-323.

43. Jermendy G, Nadas J, Hegyi I, et al. Assessment of cardiometabolic risk among shift workers in Hungary. Health Qual Life Outcomes 2012, 10:18.

44. Esquirol Y, Perret B, Ruidavets JB, et al. Shift work and cardiovascular risk factors: new knowledge from the past decade. Arch Cardiovasc Dis 2011, 104:636-668.

45. Hermida RC, Ayala DE, Mojon A, et al. Bedtime dosing of antihypertensive medications reduces cardiovascular risk in CKD. J Am Soc Nephrol 2011, 22:2313-2321.

46. Zhao $P$, Xu P, Wan $C$, et al. Evening versus morning dosing regimen drug therapy for hypertension. Cochrane Database Syst Rev 2011:CD004184. 


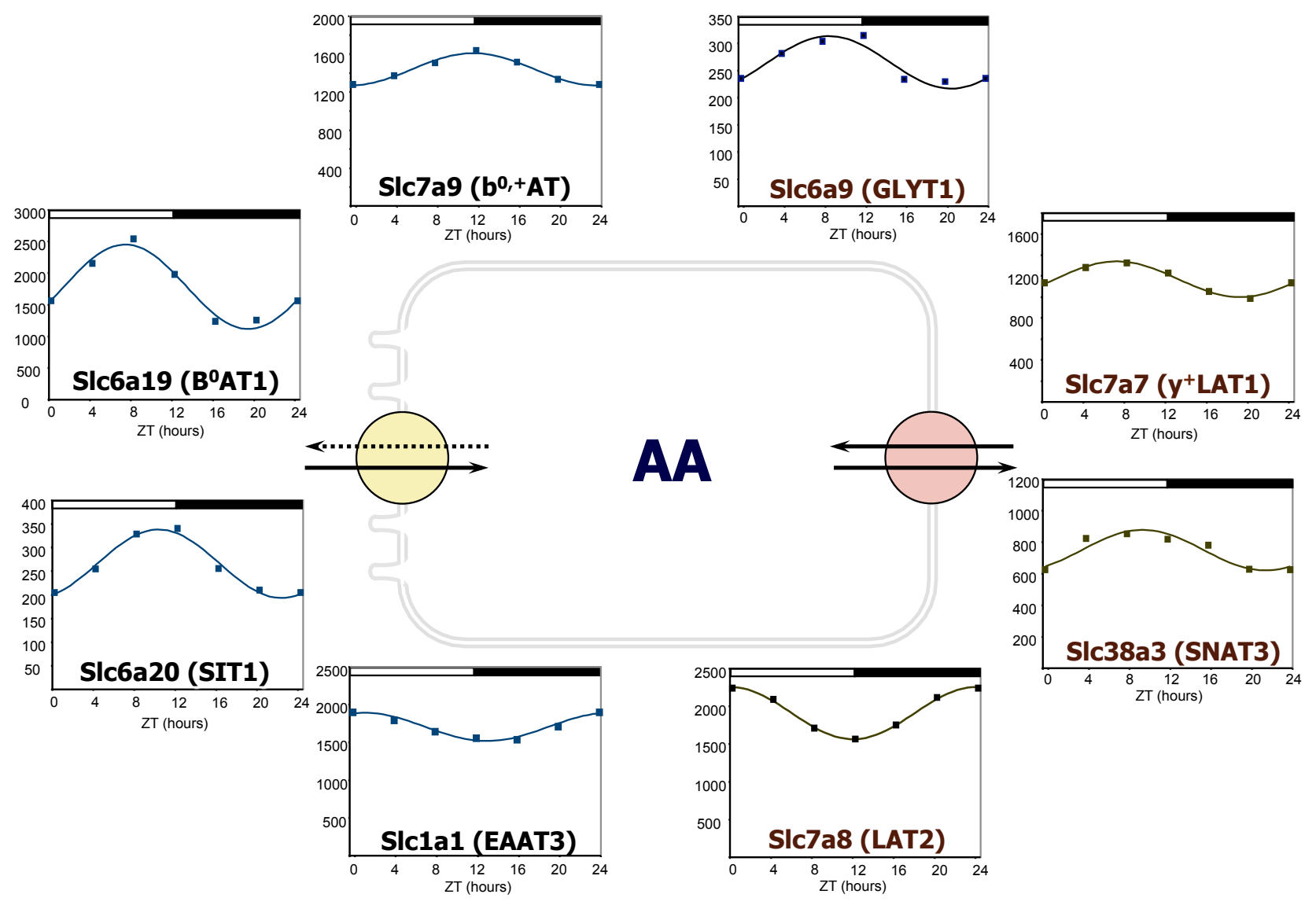

Figure 1. Bonny \& Firsov 


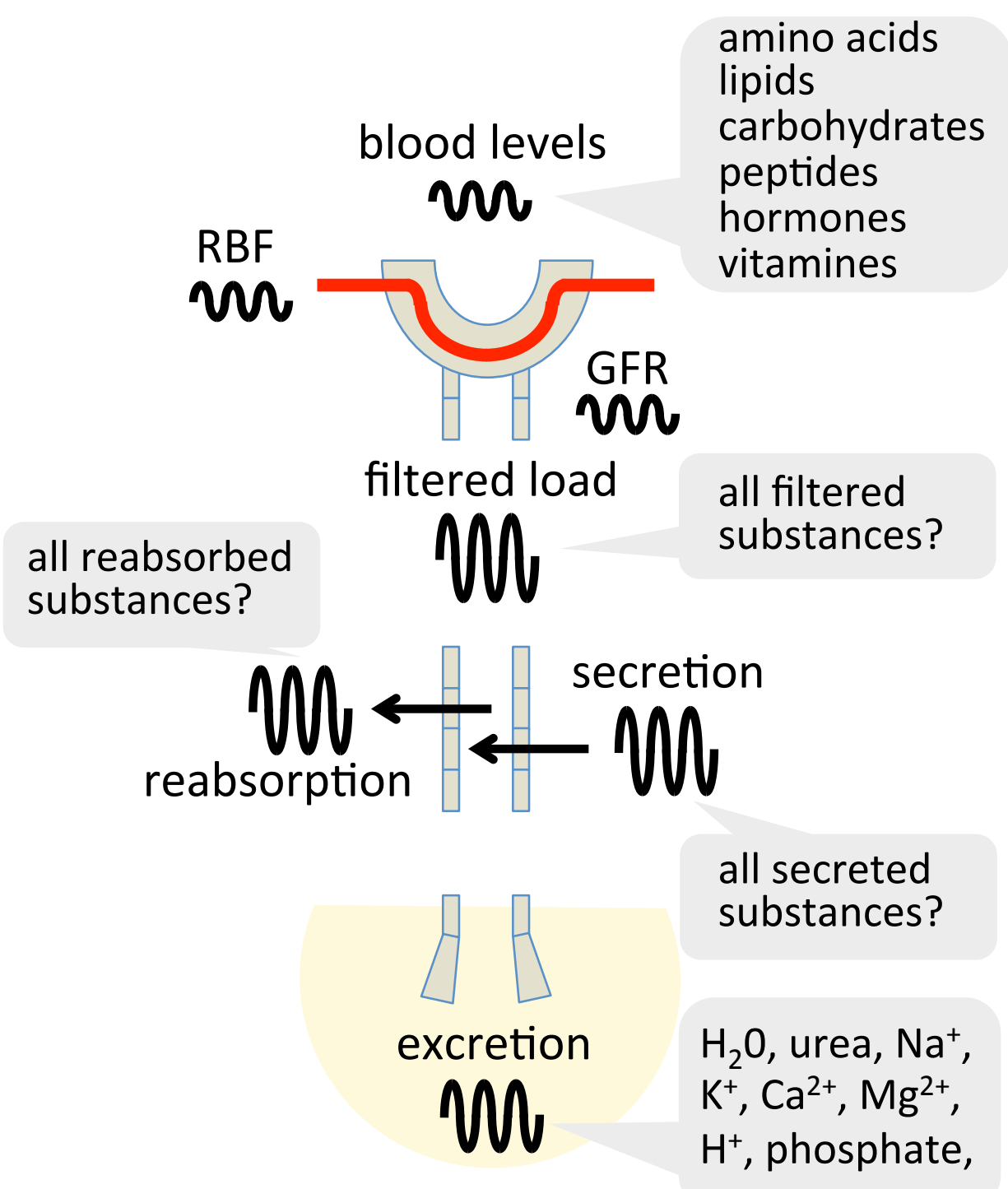

Figure 2. Bonny \& Firsov 